\title{
A Method for Attitude Control Based on a Mathematical Model for an Inverted Pendulum-Type Mobile Robot
}

\author{
Jin-Ho Yoon*, Myung-Jin Chung** \\ * Department of New Technology Convergence, Korea Polytechnic University, Korea \\ ** Department of Mechatronics Engineering, Korea Polytechnic University, Korea
}

\begin{tabular}{l} 
Article Info \\
\hline Article history: \\
Received Aug 21, 2015 \\
Revised Nov 10, 2015 \\
Accepted Nov 29, 2015 \\
\hline
\end{tabular}

Keyword:

Attitude control

Disturbance

Inverted pendulum

Mathematical model

Mobile robot

PID controller

\begin{abstract}
Amethod for attitude control based on a mathematical model for an inverted pendulum-type mobile robot was proposed. The inverted pendulum-type mobile robot wasdesigned and the mathematical modeling was conducted. The parameters of the mobile robot were estimated and the state-space model of mobile robot was obtained by the substitution of the estimated parameters into the mathematical model. The transfer functionof the mobile robot is applied to generate the root-locus diagram used for the estimation of the gains of the PID controller. The attitude control method including a PID controller, non-linear elements, and integral saturation prevention was designed and simulated. The experiment was conducted by applying the method to the mobile robot. In the attitude control experiment, the performance of attitude recovery from $\pm 12^{\circ}$ tilted initial statewith a settling time of $0.98 \mathrm{~s}$ and a percent overshoot of $40.1 \%$ was obtained. Furthermore, the attitude maintaining robustness against disturbance wasverified.
\end{abstract}

Copyright (c) 2016 Institute of Advanced Engineering and Science. All rights reserved.

\section{Corresponding Author:}

Myung-Jin Chung,

Department of Mechatronics Engineering,

Korea Polytechnic University,

237, Sangidaehak-Ro, Siheung-Si, Gyeonggi-Do, Korea Polytechnic University, Republic of Korea.

Email: mjchung@kpu.ac.kr

\section{INTRODUCTION}

In recent times, several platforms for mobile robot have been proposed by many manufacturing companies. The PUMA, which is a vehicle-type mobile robot for short distance transportation, is developed by Segway. The TBot, developed by IHMC, is an inverted pendulum-type mobilerobot for military applications and is a reconfigurable robot that can switch between a four-wheeled and a two-wheeled configuration. As an industrial service robot, the EMIEW1, having a humanoid-type upper body and a twowheel-type lower body, and the EMIEW2 having a humanoid-type body are proposed by Hitachi. In recent times, numerous researches has being conducted on inverted pendulum-type two-wheeled mobile robots because of their advantages such as low power consumption, light weight, simple configuration, excellent expandability, and a wide area of applications [1], [2], [3], [4], [5]. Since the inverted pendulum-type mobile robot has unstable characteristics in the system configuration, high technologies such as precision attitude sensing and control algorithm are required for robust attitude balance [6], [7], [8]. Furthermore, a combined design including the mechanism, hardware, and software is required [9].

In this study, an attitude control method based on a mathematical model for an inverted pendulumtype mobile robot is proposed. The inverted pendulum-type mobile robot was designed and parameters were estimated by mathematical modeling. The state-space model for mobile robot was obtained by the substitution of the estimated parameters into the mathematical model. The transfer function of mobile robot is applied to generate the root-locus diagram used for the estimation of the gains of the PID controller. The attitude control method including a PID controller, non-linear elements, and integral saturation prevention 
wasdesigned and simulated. The experiment was conducted by applying the method to the mobile robot. In the attitude control experiment, the performance of attitude recovery from $\pm 12^{\circ}$ tilted initial state and the attitude maintaining robustness of the robot against disturbance was verified.

\section{CONFIGURATION OF INVERTED PENDULUM TYPE MOBILE ROBOT}

The configuration of an inverted pendulum-type mobile robotis as follows: (Figure 1), a sensing modulefor the sensing the attitude of the body, a moving module for moving the body and generating the inertia force, a control module for controlling the attitude balance, and a communication module for transferring the data between the mobile robot and the main computer.

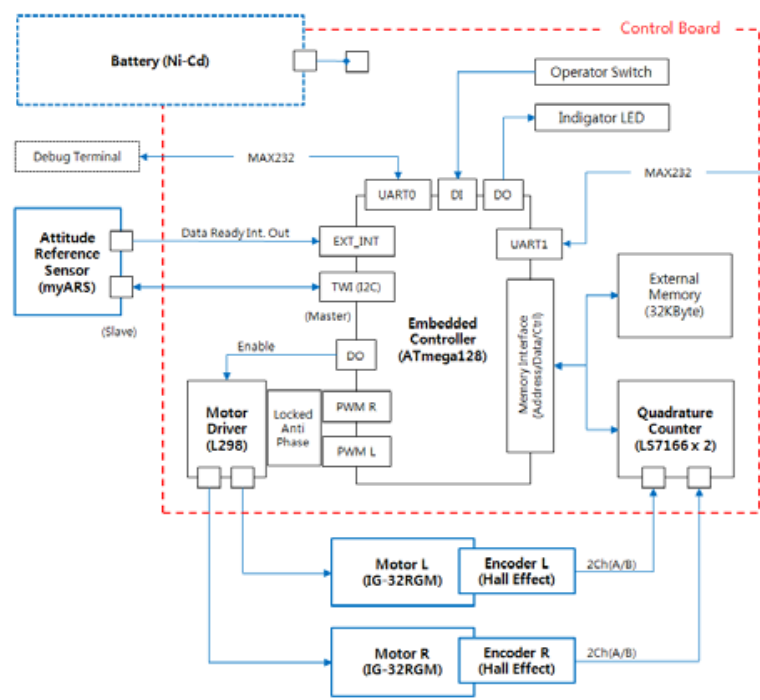

(a)

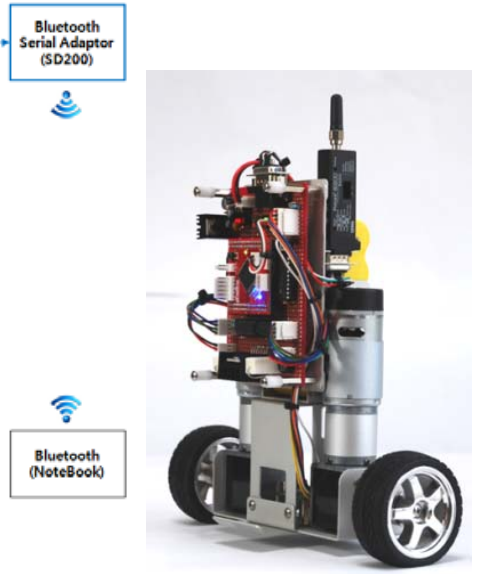

(b)

Figure 1. Configuration of an inverted pendulum-type mobile robot; (a) control diagram, (b) robot platform

\section{MATHEMATICAL MODEL}

The mathematical model of the inverted pendulum-type mobile robot is obtained as the state-space model with two degrees of freedom using the free-body and the kinematic diagram as shown in Figure 2.

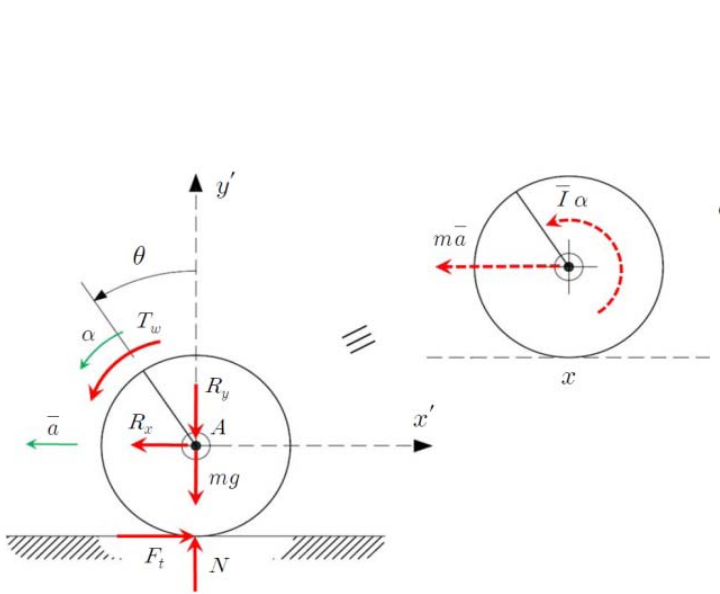

(a)

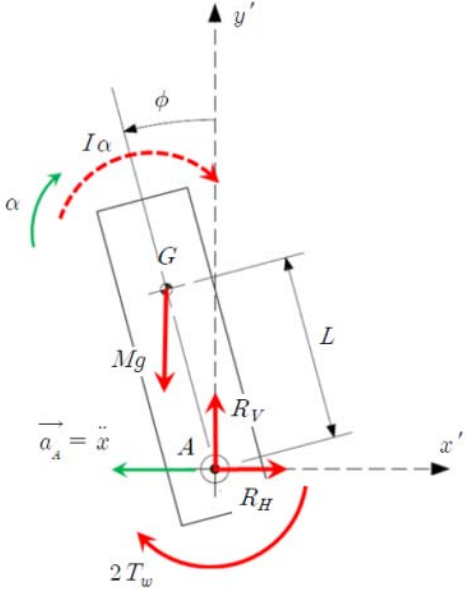

(b)

Figure 2. Free-body and kinematic diagram of inverted pendulum-type mobile robot; (a) wheel, (b) body 
From Figure 2(a), the equation for the motion of the wheel is obtained using the horizontal and the rotational components as described in equation (1) [8].

$$
2\left(m r^{2}+I_{\omega}\right) \ddot{\theta}=2 T_{\omega}+R_{H} r
$$

Where, $m$ is the mass, $r$ is theradius, $I_{w}$ is the mass moment of inertia, and $\theta$ is the angle of rotation of the wheel, respectively. $T_{w}$ is the torque generated by the wheel motor and $R_{H}$ is the sum of the horizontal components of the reaction force between the two wheels and the body. It is assumed that the wheel has only a rotational motion without a sliding motion.

From Figure 2(b), the equation for the motion of the body is obtained using the horizontal and the rotational components as described in equation (2).

$$
\left(I_{p}+M L^{2}\right) \ddot{\phi}+M r L \ddot{\theta} \cos \phi-M g L \sin \phi=-2 T_{\omega}
$$

Where, $I_{p}$ is the mass moment of inertia, $M$ is the mass, and $\varphi$ is the angle of rotation of the body, respectively. $\mathrm{L}$ is the distance between the center of mass of the wheels (A) and the center of mass of the $\operatorname{body}(\mathrm{G})$.

From Figure 2(a) and $2(\mathrm{~b}), \mathrm{R}_{\mathrm{H}}$ is obtained using the horizontal and rotational motion of the wheel and body as described in equation (3).

$$
R_{H}=-M r \ddot{\theta}-M L \cos \phi \ddot{\phi}+M L \sin \phi \dot{\phi}^{2}
$$

By substituting the equation (3) in equations (1) and (2), and linearizing at the equilibrium point $\varphi=$ 0 , the equations of motion for the wheel and the body are simplified as follows:

$$
\begin{aligned}
& \left.\left[(2 m+M) r^{2}+2 I_{\omega}\right)\right] \ddot{\theta}+M r L \ddot{\phi}=2 T_{\omega} \\
& \left(I_{p}+M L^{2}\right) \ddot{\phi}+M r L \ddot{\theta}-M g L \phi=-2 T_{\omega}
\end{aligned}
$$

In equation (4) and (5), wheel torque $\left(\mathrm{T}_{\mathrm{w}}\right)$ can be described using the equivalent circuit of the wheel motor as shown in Figure 3.

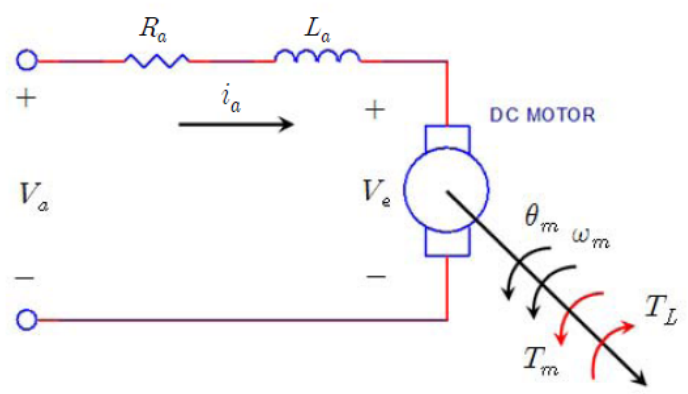

Figure 3. Equivalent circuit of the DC motor

By ignoring inductance and the coefficient of friction of the motor, applying Kirchhoff's voltage law, and using the equation for the moment at the axis of the motor, the load torque is calculated as follows:

$$
T_{L}=-\frac{K_{T} K_{b}}{R_{a}} \omega_{m}+\frac{K_{T}}{R_{a}} V_{a}-J_{m} \ddot{\theta_{m}}
$$


Where, $\mathrm{K}_{\mathrm{T}}$ is the torque constant, $\mathrm{K}_{\mathrm{b}}$ is the back emf constant, $\mathrm{R}_{\mathrm{a}}$ is the resistance, $\mathrm{V}_{\mathrm{a}}$ is the applied voltage, and $\mathrm{J}_{\mathrm{m}}$ is the moment of inertia of the motor.

When the gear ratio is $n$, the wheel torque $\left(T_{w}\right)$ and the load torque $\left(T_{L}\right)$ are related by the equation $\mathrm{T}_{\mathrm{w}}=\mathrm{nT}_{\mathrm{L}}$. The angular displacement of the motor $\left(\theta_{\mathrm{m}}\right)$ and the angular displacement of the wheel $(\theta)$ are related by the equation $\theta=\left(\theta_{\mathrm{m}} / \mathrm{n}\right)+\varphi$, where the initial angular displacement is $\varphi$. By substituting these relations in equation (6), the wheel torque is described by the following equation:

$$
T_{\omega}=-\frac{K_{T} K_{b}}{R_{a}} n^{2} \dot{\theta}+\frac{K_{T} K_{b}}{R_{a}} n^{2} \dot{\phi}+n \frac{K_{T}}{R_{a}} V_{a}-J_{m} n^{2} \ddot{\theta}+J_{m} n^{2} \ddot{\phi}
$$

By substituting the equation (7) in equations (4) and (5), the equations of motion are completed. The state-space model is obtained by applying the system parameters, which are calculated and measured [7], as described in equation (8).

$$
\begin{aligned}
& X=A X+B u, \\
& Y=C X, \\
& X=[\theta \dot{\theta} \dot{\phi} \dot{\phi}]^{T}, \\
& u=V_{a} .
\end{aligned}
$$

Where we have:

$$
A=\left[\begin{array}{cccc}
0 & 1 & 0 & 0 \\
0 & -13.41 & 46.63 & 13.41 \\
0 & 0 & 0 & 1 \\
0 & 4.45 & 50.82 & -4.45
\end{array}\right], B=\left[\begin{array}{c}
0 \\
14.14 \\
0 \\
-4.69
\end{array}\right], \text { and } C=\left[\begin{array}{llll}
1 & 0 & 0 & 0 \\
0 & 1 & 0 & 0 \\
0 & 0 & 1 & 0 \\
0 & 0 & 0 & 1
\end{array}\right]
$$

\section{CONTROL METHOD AND EXPERIMENT}

Although the state-space model has two degrees of freedom, the state-space equation is converted tothe transfer function as given in equation (9), and the gains of the PID controller can be estimated using the root-locus diagram generated from this transfer function.

$$
G(s)=\frac{\Phi(s)}{V_{a}(s)}=\frac{4.69 s}{s^{3}+17.86 s^{2}-50.82 s-889.2}
$$

The mobile robotis unstable because it has the poles on the right plane as shown in Figure 4(a). Using the root-locus diagram, the range of the proportional gain for the stability of the system can be estimated in the different cases where the PI or the PID controlleris used (Figure 4). 


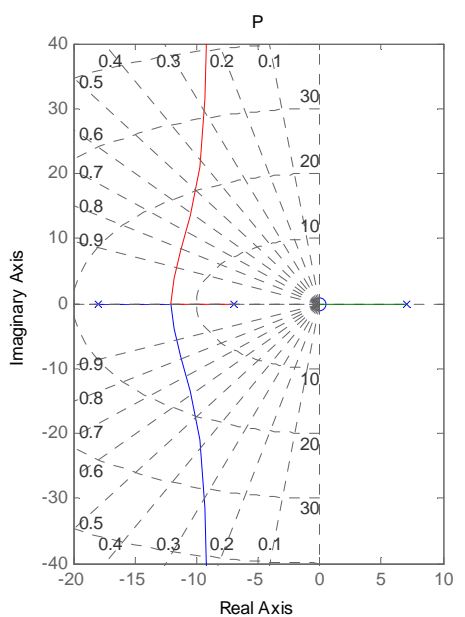

(a)

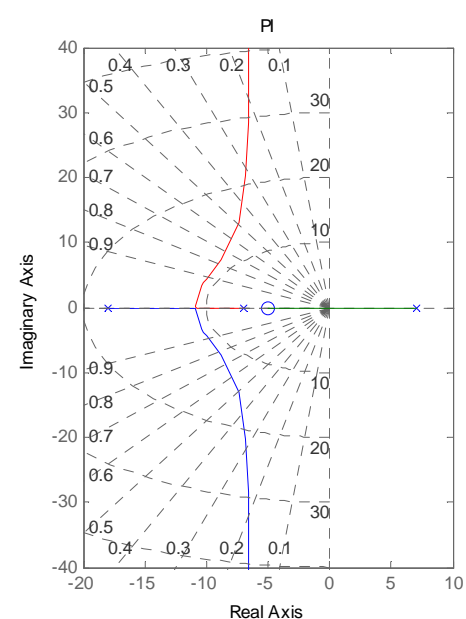

(b)

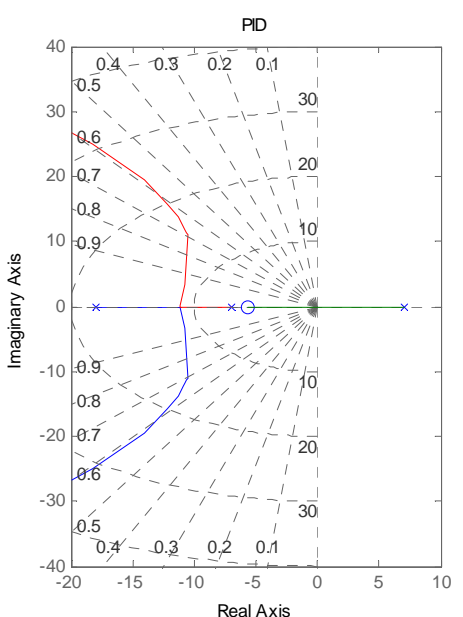

(c)

Figure 4. Root-locus diagram for the mobile robot according to the type of controller used; (a) P controller, (b) PI controller, (c) PID controller

Root-locus diagram of the mobile robot can be changed by added zeros and poles using the PI or PID controller as shown in Figures 4(b) and 4(c). Equations (10) and (11) describe the transfer function of the PI and PID controller. From these equations, the positions of the zeros can be determined by the gain of the controller.

$$
\begin{aligned}
& G_{P I}(s)=\frac{K_{p}\left(s+K_{i} / K_{p}\right)}{s} \\
& G_{P I D}(s)=\frac{K_{d}\left(s^{2}+K_{p} / K_{d} s+K_{i} / K_{d}\right)}{s}
\end{aligned}
$$

Where, $\mathrm{K}_{\mathrm{p}}$ is the propotional gain, $\mathrm{K}_{\mathrm{i}}$ is the integral gain, and $\mathrm{K}_{\mathrm{d}}$ is the derivative gain.

A transfer function model cannot reflect the initial state of the tilted body. By applying the statespace model and the feedback from the state variables, the attitude control system including a PID controller, non-linear elements, and integral saturation prevention is constructed in the Simulink as shown in Figure 5.

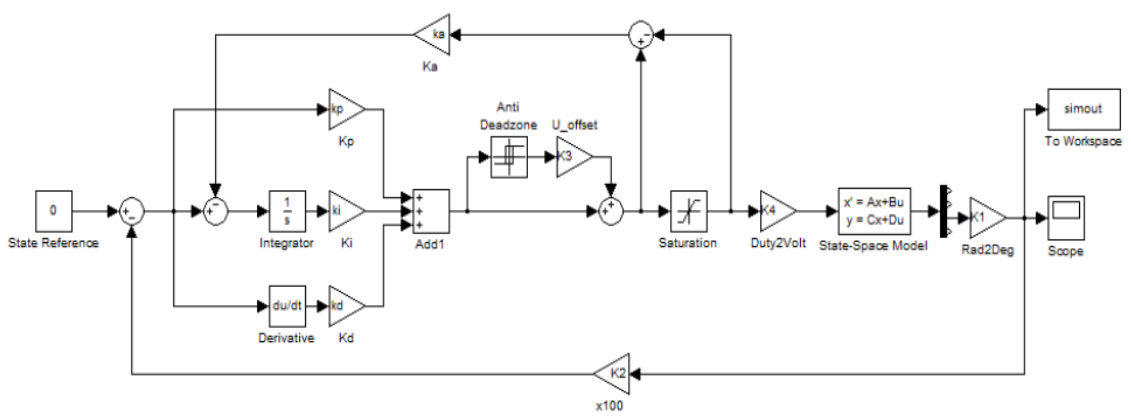

Figure 5. Attitude control system constructed in the Simulink

In Figure 4, the proportional gain is estimated to be 200 for a percent overshoot of $30 \%$. The integral and derivative gains are determined by the simulation of the root-locus diagram based on the estimated proportional gain. Using the estimated gain parameters, the performance of the attitude control systemis simulated with and without the adoption of the non-linear elements as shown in Figure 6. 


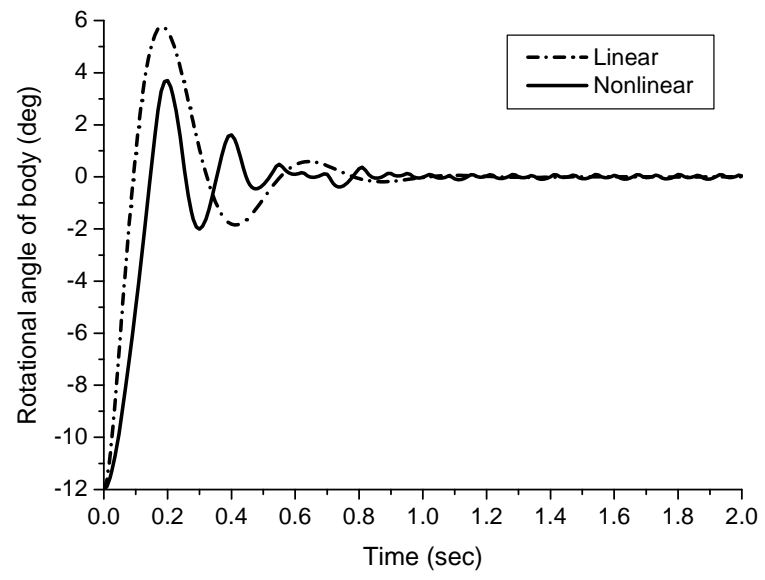

Figure 6. Simulation result of attitude control system with and without the non-linear elements

The experiment is conducted by applying the developed attitude control system having the estimated control gain to the mobile robot. The initial state of the mobile robot is tilted by $\pm 12^{\circ}$ and the angle is reduced and maintained at zero by attitude control. Figure 7 shows the performance of attitude control using the PID gains obtained by simulation including the compensationfor disturbance. The gains of $\mathrm{K}_{\mathrm{p}}, \mathrm{K}_{\mathrm{i}}$, and $\mathrm{K}_{\mathrm{d}}$ are 1.1, 17 , and 0.01 respectively. Table 1 lists the performance of attitude control determined from the experiment and simulation.

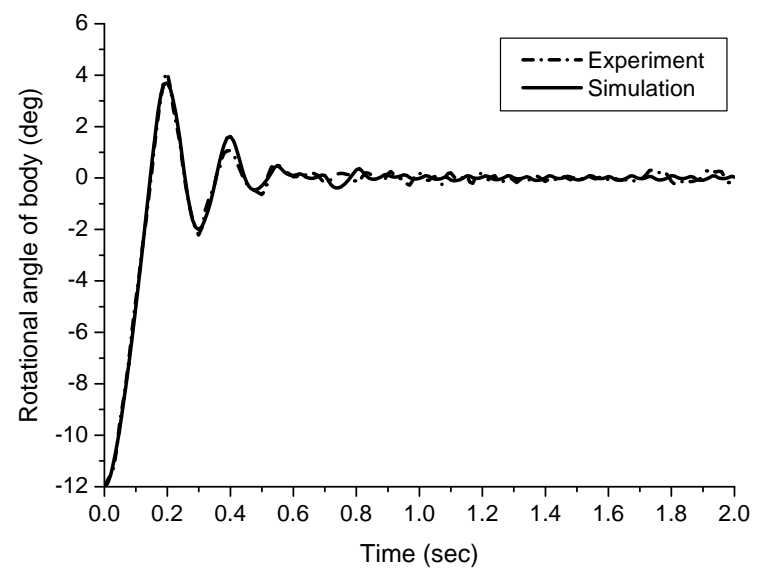

Figure 7. Attitude control performance using the PID gainsobtained from simulation

Table 1. Performance of attitude control

\begin{tabular}{ccccc}
\hline Variable & Rising Time $(\mathrm{s})$ & $\% \mathrm{OS}(\%)$ & Settling Time $(\mathrm{s})$ & Steady State Error $(\mathrm{deg})$ \\
\hline Experiment & 0.14 & 40.1 & 0.98 & \pm 0.3 \\
Simulation & 0.13 & 37.0 & 0.83 & \pm 0.1 \\
\hline
\end{tabular}

\section{CONCLUSION}

The attitude control method based on a mathematical model for an inverted pendulum-type mobile robot was proposed. The inverted pendulum-type mobile robot was designed and the mathematical modeling was conducted. The parameters of mobile robot were estimated and the state-space model of mobile robot was obtained by the substitution of the estimated parameters into the mathematical model. The transfer function of mobile robot is applied to generate the root-locus diagram used for the estimation of the gains of the PID controller. The attitude control method including a PID controller, non-linear elements, and integral saturation prevention was designed and simulated. The experiment was conducted by applying the system to 
the mobile robot. In the attitude control experiment, the performance of attitude recovery from $\pm 12^{\circ}$ tilted initial state with a settling time of $0.98 \mathrm{~s}$ and a percent overshoot of $40.1 \%$ was obtained. Furthermore, the attitude maintaining robustness against disturbance was verified.

\section{ACKNOWLEDGEMENTS}

This work was supported by the National Research Foundation of Korea Grant funded by the Korean Government (31Z20130012987).

\section{REFERENCES}

[1] F. Grasser, et al, "JOE: A Mobile, Inverted Pendulum", IEEE Transactions on Industrial Electronics, vol. 49, pp. 107-114, 2002.

[2] T.S. Jin, "Command Fusion for Navigation of Mobile Robots in Dynamic Environments with Objects", Journal of information and communication convergence engineering, vol. 11, pp. 24-29, 2013.

[3] D.L. Nguyen and M.E. Lee, "OSEK/VDX Porting to the Two-Wheel Mobile Robot Based onthe Differential Drive Method", Journal of information and communication convergence engineering, vol. 10, pp. 372-377, 2012.

[4] Y.H. Kim, et al, "Dynamic Analysis of a NonholonomicTwo-Wheeled Inverted Pendulum Robot", Journal of Intelligent and Robotic Systems, vol. 44, pp. 25-46, 2005.

[5] H.N. Ha, and J.M. Lee, "A Control of Mobile Inverted Pendulum using Single Accelerometer", Journal of Institute of Control, Robotics and Systems, vol. 16, pp. 440-445, 2010.

[6] M. Sudarma, et al, "Design and Implementations of Control System QuadrupedRobot Driver Application Based on Windows Platform", International Journal of Electrical and Computer Engineering, vol. 5, pp. 251-258, 2015.

[7] J.H. Yoon, et al, "Mathematical Model Based Attitude Control of Inverted Pendulum Type Mobile Robot", Applied Mechanics and Materials, vol. 789-790, pp. 698-702, 2015.

[8] J.H. Yoon, et al, "Attitude Control Method of Inverted Pendulum Type Mobile Robot", in International Conference on Future Information \& Communication Engineering, ICFICE 2015, 2015, pp. 557-560.

[9] K.H. Choi, "Optimal Design and Control of Mobile Inverted Pendulum Robot", Master's Thesis of Mechanical Engineeringin Korea Polytechnic University, 2012.

\section{BIOGRAPHIES OF AUTHORS}

Jin-Ho Yoon received his B.S. in mechatronics engineering (2014) from the Korea Polytechnic University in Korea, where heis currently pursuing the M.S. in new technology convergence engineering

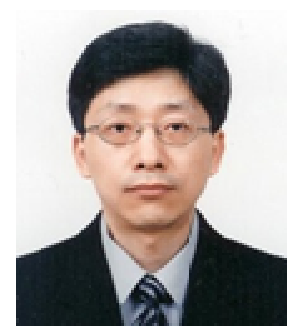

Myung-Jin Chung received his M.S. in production engineering (1991) and a $\mathrm{PhD}$ in mechanical engineering (2002) from the Korea Advanced Institute Science and Technology (KAIST). Now he is a professor in the mechatronics engineering department of the Korea Polytechnic University in Korea. His current research interests include the control of quadcopter, inverted pendulum-type mobile robots, high-precision positioning systems, and semiconductor manufacturing systems. 